\title{
Nonequilibrium dynamics of bosonic Mott insulators in an electric field
}

\author{
M. Kolodrubetz, ${ }^{1}$ D. Pekker, ${ }^{2}$ B. K. Clark, ${ }^{1,3}$ and K. Sengupta ${ }^{4}$ \\ ${ }^{1}$ Department of Physics, Princeton University, Princeton, New Jersey 08544, USA \\ ${ }^{2}$ Department of Physics, California Institute of Technology, Pasadena, California 91125, USA \\ ${ }^{3}$ Princeton Center for Theoretical Science, Princeton University, Princeton, New Jersey 08544, USA \\ ${ }^{4}$ Theoretical Physics Department, Indian Association for the Cultivation of Science, Jadavpur, Kolkata-700032, India
}

(Received 2 February 2012; published 16 March 2012)

\begin{abstract}
We study the nonequilibrium dynamics of one-dimensional Mott-insulating bosons in the presence of a tunable effective electric field $\mathcal{E}$ which takes the system across a quantum critical point separating a disordered and a translation symmetry broken ordered phase. We provide an exact numerical computation of the residual energy $Q$, the $\log$ fidelity $F$, the defect density $D / L$, and the order parameter correlation function for a linear-in-time variation of $\mathcal{E}$ with a rate $v$. We discuss the temporal and spatial variation of these quantities for a range of $v$ and for finite system sizes as relevant to realistic experimental setups [J. Simon et al., Nature (London) 472, 307 (2011)]. We show that in finite-sized systems $Q, F$, and $D$ obey Kibble-Zurek scaling, and suggest further experiments within this setup to test our theory.
\end{abstract}

DOI: 10.1103/PhysRevB.85.100505

PACS number(s): 67.85.-d, 75.10.Jm, 64.70.Tg

Universality is one of the central concepts of physics. While in equilibrium it has long been known that systems that share the same universality class also share critical exponents, this is less clear with nonequilibrium dynamics. As integrability has a strong influence on dynamical phenomena, of particular concern is whether systems that share the same universality class but differ in this aspect recover the same critical exponents. Indeed, recent evidence in Ref. 1 suggests that the dynamical exponents for a nonintegrable model differ from the expected Kibble-Zurek ones seen in integrable models. $^{2}$

Ultracold atoms in optical lattices provide us with a unique opportunity to study both equilibrium phases and nonequilibrium quantum dynamics of strongly coupled bosonic systems near a quantum phase transition (QPT). ${ }^{3}$ One system, which has been the subject of a recent experimental study, consists of one-dimensional (1D) Mott-insulating (MI) bosons in the presence of an effective electric field $\mathcal{E}$. ${ }^{4}$ It has been shown that this system can be described in terms of an effective quantum dipole model or, equivalently, an Ising spin model with infinitely strong nearest-neighbor coupling in the presence of both a transverse and a longitudinal field. ${ }^{5}$ Furthermore, at zero temperature, tuning $\mathcal{E}$ to a critical value $\mathcal{E}_{c}$ leads to a QPT. ${ }^{5}$ In the dipole language, this transition consists of a change in the ground state from a dipole vacuum (which corresponds to $\bar{n}$ bosons at each site) to one with maximal dipoles (which corresponds to alternating $\bar{n}-1$ and $\bar{n}+1$ bosons per site). In the spin language, this transition is from the paramagnet (PM) to an Ising antiferromagnet (AFM). The intermediate quantum critical point (QCP) belongs to the Ising universality class. ${ }^{5}$ The appearance of this AFM order has recently been observed using a quantum gas microscope. ${ }^{4}$ Theoretical studies of the phases of the bosonic Mott insulator in an electric field have also been extended to several two-dimensional (2D) lattices. $^{6}$

The study of nonequilibrium dynamics of closed quantum systems has seen tremendous progress in recent years. ${ }^{2}$ One reason for this intense effort has been the possibility of experimentally realizing these dynamics using ultracold atoms.
Indeed, experiments probing nonequilibrium phenomena with strongly coupled 2D bosonic atoms, well described by the 2D Bose-Hubbard model, have been carried out recently. ${ }^{7}$ The corresponding theoretical studies show a reasonable qualitative match with experiments. ${ }^{8}$ For the case of 1D bosonic MI in the presence of an electric field, the dynamics following sudden quenches of the electric field have also been studied theoretically. ${ }^{9-12}$ However, to the best of our knowledge, the case of finite velocity and, in particular, nearly adiabatic ramps of $\mathcal{E}$ has not been previously explored.

In this Rapid Communication, we probe the nonequilibrium quantum dynamics of the bosonic Mott insulators in the presence of a linear-in-time varying electric field (chemical potential gradient)

$$
\mathcal{E}(t)=\mathcal{E}_{i}+\left(\mathcal{E}_{f}-\mathcal{E}_{i}\right) t / \tau,
$$

where $\mathcal{E}_{i}$ and $\mathcal{E}_{f}$ are the initial and final values of the electric field, and $\tau$ is the ramp time. We define the ramp rate to be $v=\partial_{t} \mathcal{E}(t)$. We look at ramps that start from the PM phase with unit boson occupation per site and end either in the AFM phase across the QPT or at the QCP. Based on the resonant manifold model of Ref. 5, we provide an exact numerical computation for finite system sizes $(L)$, using exact diagonalization $(\mathrm{ED}, L \leqslant 16)$ and time-dependent matrix product states (tMPS, $L \leqslant 96$ ), of the residual energy $Q$, the $\log$ fidelity $F$, the number of dipoles $n_{d}$, and the dipole correlation function $C_{i j}(t)$. We note that the experimental setup of Ref. 4 constitutes systems of $L \lesssim 50$ lattice sites and measures $n_{d}$ as well as $C_{i j}(t)$; hence our theoretical analysis constitutes a quantitatively exact description of the dynamics of the experimental system providing a valuable guideline to future experiments. ${ }^{13}$ Further, for finite-sized systems, our analysis reveals the manifestation of universal KibbleZurek-like (KZ) scaling with Ising exponents. ${ }^{14-16}$ In the past, KZ scaling has been shown to work well in integrable systems $;{ }^{17}$ however, its manifestation in nonintegrable systems has not been consistently demonstrated. ${ }^{1}$ Our work thus provides a realization of $\mathrm{KZ}$ scaling in finite-sized nonintegrable systems which can be tested with an existing experimental 


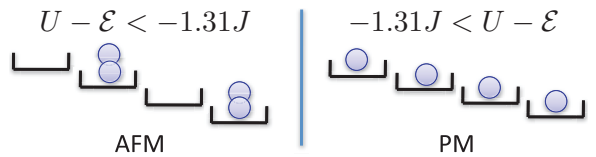

FIG. 1. (Color online) A pictorial representation of the dipole (AFM) and the vacuum (PM) ground states for MI bosons with $\bar{n}=1$ across the quantum phase transition. The transition occurs at $U-$ $\mathcal{E}_{c}=-1.31($ for $J=1)$.

setup. Furthermore, we investigate the crossover from LandauZener (LZ) scaling ${ }^{15,18}$ to KZ scaling in finite-sized systems, and show that it collapses onto a universal functional form, as suggested in Ref. 19.

Model. Bosonic atoms in a tilted optical lattice (i.e., in an effective time-dependent electric field) are well described using the Bose-Hubbard Hamiltonian ${ }^{5}$

$$
H_{B}(t)=-t_{0} \sum_{\langle i j\rangle} b_{i}^{\dagger} b_{j}+\frac{U}{2} \sum_{i} n_{i}\left(n_{i}-1\right)-\mathcal{E}(t) \sum_{i} i \cdot n_{i},
$$

where $\langle i j\rangle$ denotes the sum over nearest neighbors, $b_{i}$ the boson annihilation operator at site $i, n_{i}=b_{i}^{\dagger} b_{i}$ the boson number operator, $t_{0}$ the hopping amplitude, and $U$ the on-site interaction. Under the condition $U, \mathcal{E}(t) \gg|U-\mathcal{E}(t)|, t_{0}$, the low-energy dynamics of $H_{B}$ are captured by an effective dipole model $^{5}$

$$
H_{d}(t)=[U-\mathcal{E}(t)] \sum_{\ell} d_{\ell}^{\dagger} d_{\ell}-J \sum_{\ell}\left(d_{\ell}^{\dagger}+d_{\ell}\right),
$$

where $d_{\ell}=b_{i} b_{j}^{\dagger} / \sqrt{n_{0}\left(n_{0}+1\right)}$ denotes the dipole annihilation operator which lives on a link $\ell$ between the neighboring sites $i$ and $j$ as schematically shown in Fig. 1, $n_{0}$ the boson occupation of the parent Mott insulator, and $J=$ $t_{0} \sqrt{n_{0}\left(n_{0}+1\right)}$ the amplitude for creation or annihilation of a dipole. Henceforth, we shall use the units in which $\hbar=1$, $J=1$, and restrict ourselves to $n_{0}=1$. The dipoles satisfy two constraints: First, there can be only one dipole on any link $\ell$ which renders $d_{\ell}^{\dagger} d_{\ell} \leqslant 1$, and second, two consecutive links can not be simultaneously occupied by dipoles $d_{\ell}^{\dagger} d_{\ell+1}^{\dagger} d_{\ell+1} d_{\ell}=0$. These constraints render $H_{d}$ nonintegrable; however, they also lead to a significant reduction in the Hilbert space of $H_{d}$ which makes ED and tMPS the methods of choice for studying the dynamics of the model. In addition, we restrict ourselves to studying $H_{d}$ with periodic boundary conditions so as to approach the thermodynamic limit with smaller systems. We note that the dipole model can be represented in terms of an Ising-like spin model via the transformation $S_{\ell}^{z}=1 / 2-d_{\ell}^{\dagger} d_{\ell}, S_{\ell}^{x(y)}=(-i)\left[d_{\ell}+(-) d_{\ell}^{\dagger}\right] / 2.4,5$ Note that $(-1)^{\ell}\left\langle S_{\ell}^{z}\right\rangle$ is the order parameter for the transition from the PM (dipole vacuum) to the AFM (maximal dipole density) state.

To study the dynamics within exact diagonalization, we evolve the wave function using the time-dependent Hamiltonian of Eq. (2), in which the electric field is tuned linearly in time according to Eq. (1),

$$
i \hbar \partial_{t}|\psi(t)\rangle=H_{d}(t)|\psi(t)\rangle .
$$

We supplement the time evolution with the initial condition $|\psi(t=0)\rangle=\left|\psi_{G}\right\rangle_{i}$, where $\left|\psi_{G}\right\rangle_{i}$ is the ground-state wave function of the initial Hamiltonian. Integrating Eq. (3) from $t=0$ to $t=\tau$ we obtain the wave function at the end of the $\operatorname{ramp}|\psi(\tau)\rangle$.

We supplement our exact diagonalization studies by tMPS, which allows us to study larger system sizes. tMPS represents the wave function as $|\psi\rangle=\sum_{\left\{\sigma_{i}\right\}} M_{1}^{\sigma_{1}} M_{2}^{\sigma_{2}} \ldots M_{L}^{\sigma_{L}}\left|\sigma_{1}, \sigma_{2} \ldots\right\rangle$, where $M_{i}^{\sigma_{i}}$ are a set of $\chi$ by $\chi$ matrices indexed by site $i$ and spin $\sigma_{i}$ (except $M_{1}$ and $M_{L}$, which are row and column vectors respectively). ${ }^{20}$ We take advantage of the fact that in the reduced Hilbert space the Hamiltonian $H_{d}(t)$, Eq. (2), is a sum of single-site Hamiltonians to perform time evolution. We evolve in time via $|\psi(t+\epsilon)\rangle=P \exp \left[-i \epsilon H_{d}(t)\right]$ by first exactly applying the single-site Hamiltonian $H_{d}(t)$ and then projecting out configurations with neighboring dipoles using the projection operator $P$. This projection increases the MPS bond dimension which is then reduced back to its original value $\chi$, introducing a small error. The method becomes exact in the limit $\epsilon \rightarrow 0$ and matrix size $\chi \rightarrow \infty$, and its convergence has been numerically checked by extrapolating in these parameters. ${ }^{21}$ Ground states are found by evolving the same Hamiltonian to large imaginary time at fixed $\mathcal{E}$.

For this work, the specific observables of interest will be the residual energy $Q$, the $\log$ fidelity $F$, the dipole number $n_{d}$ (i.e., the number of sites with even parity of the boson occupation number), the defect number $D$ (a defect, for the ramps we address, is a decrease in the number of dipoles from the ground state), and the spin-spin correlation function $C_{i j}$ which, at any instant $t$, are given by

$$
\begin{gathered}
Q(t)=\langle\psi(t)|H(t)| \psi(t)\rangle-E_{G}(t), \\
F(t)=\ln \left[\left|\left\langle\psi(t) \mid \psi_{G}(t)\right\rangle\right|^{2}\right], \\
n_{d}(t)=\left\langle\psi(t)\left|\sum_{\ell}\left(1+2 S_{\ell}^{z}\right)\right| \psi(t)\right\rangle, \\
D(t)=\left|n_{d}(t)-\left\langle\psi_{G}(t)\left|\sum_{\ell}\left(1+2 S_{\ell}^{z}\right)\right| \psi_{G}(t)\right\rangle\right|, \\
C_{i j}(t)=\left\langle\psi(t)\left|S_{i}^{z} S_{j}^{z}\right| \psi(t)\right\rangle,
\end{gathered}
$$

where $E_{G}(t)\left[\left|\psi_{G}(t)\right\rangle\right]$ corresponds to the ground-state energy (wave function) of the Hamiltonian $H_{d}(t)$. For notational brevity, we shall drop the index $t$ from observables when evaluating them at the end of the ramp $(t=\tau)$.

We begin with a discussion of $Q$ and $F$ for finite-sized systems $(L \leqslant 96)$ undergoing ramps from the PM phase $\left(U-\mathcal{E}_{i}=100\right)$ to the QCP $\left(U-\mathcal{E}_{f}=U-\mathcal{E}_{c}=-1.31\right)$ in time $\tau$. For a finite-sized system which is always gapped, $Q$ and $F$ behave differently than their counterparts in the thermodynamic limit $(L \rightarrow \infty)$ for which one expects KZ-like scaling to manifest in both $Q \sim v^{(d+z) v /(z v+1)}$ and $F \sim v^{d v /(z v+1)}$ for small $v$ (large $\tau$ ). Here $d=1$ is the dimensionality, $z=1$ is the dynamical critical exponent, and $v=1$ the correlation length critical exponent. For very fast ramps, the wave function does not have time to evolve during the dynamics, and therefore the behavior of finite- and infinite-sized systems is similar but not universal. As the ramp rate becomes slower $(v \sim 1)$, $\mathrm{KZ}$ scaling sets in for both infinite- and finite-sized systems. However, while KZ scaling is expected to persist to infinitely slow ramps in the thermodynamic limit, for finite-sized systems it is cut off for ramps slower than a critical ramp 


\section{Ramps from PM to QCP}
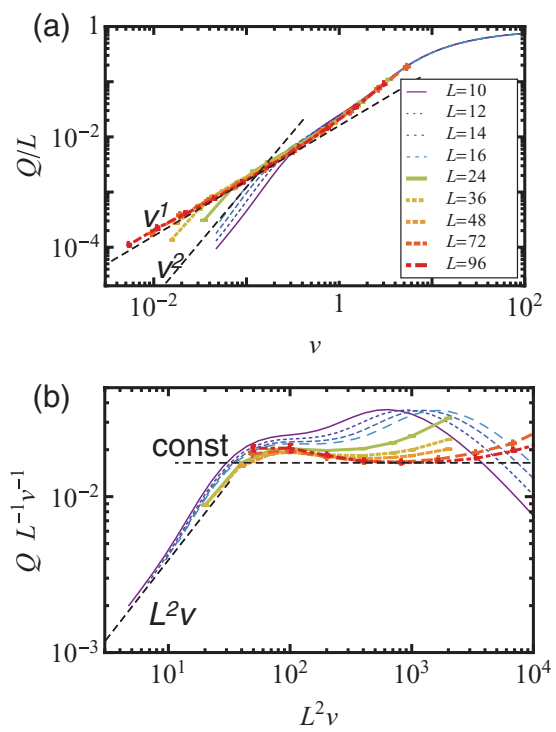
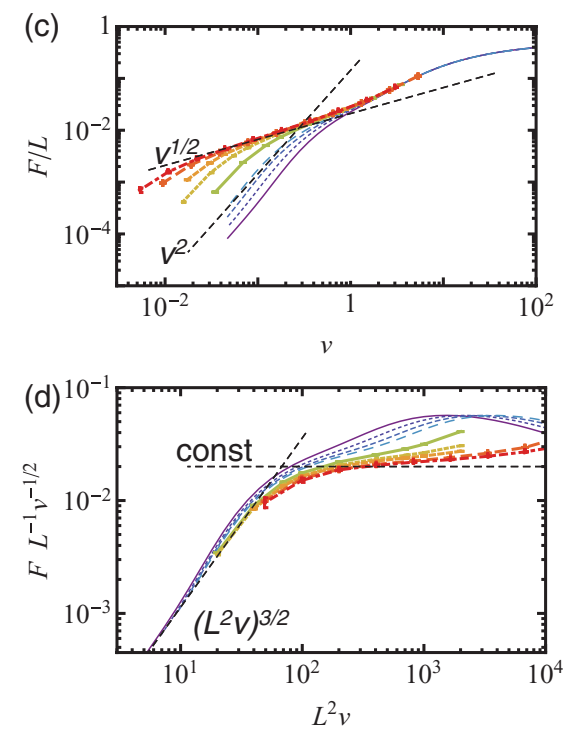

Ramps from PM to AFM
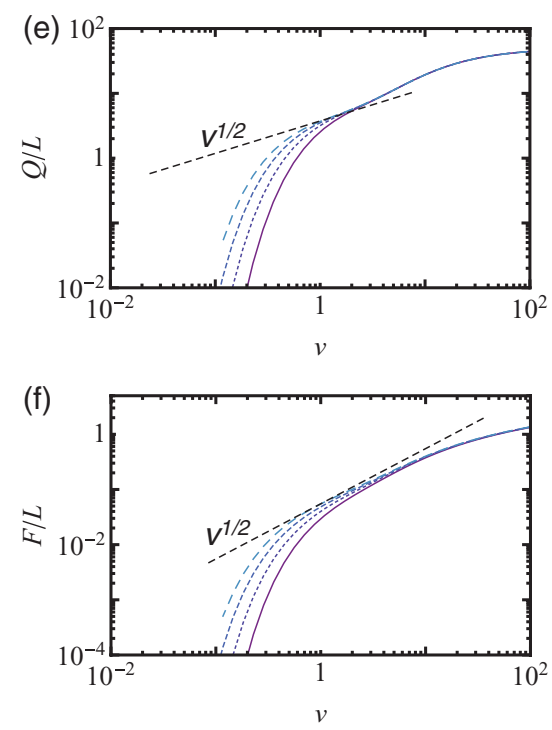

FIG. 2. (Color online) Residual energy $Q$ (a) and $\log$ fidelity $F$ (c) as a function of the ramp rate $v=\partial_{t} \mathcal{E}(t)$ for various system sizes from $10 \leqslant L \leqslant 16$ (ED) and $24 \leqslant L \leqslant 96$ (tMPS), where error bars correspond to time step extrapolation error. Bond dimension errors are below $10^{-4}$. The ramps start in the PM $\left(U-\mathcal{E}_{i}=100\right)$ and end at the QCP $\left(U-\mathcal{E}_{f}=-1.31\right)$. Dashed lines show the indicated power laws. Note the extension of the intermediate Kibble-Zurek-like scaling regime to lower values of $v$ for larger system sizes. (b) and (d) show finite-sized scaling collapse for $Q$ and $F$ for several $L$. (e) and (f) show $Q$ and $F$ as a function of $v$ for ramps starting in the PM $\left(U-\mathcal{E}_{i}=100\right)$ and ending in the AFM phase $\left(U-\mathcal{E}_{f}=-100\right)$ with $10 \leqslant L \leqslant 14$. Note the change in $Q$ power law from $v^{1}$ to $v^{1 / 2}$.

rate $v_{c}(L) \sim L^{-(1 / v+z)}$. For $v \leqslant v_{c}(L), Q$ and $F$ are expected to scale as $v^{2}$ as in gapped systems. ${ }^{15,18}$ These expectations may be formalized in the form of scaling functions

$$
\begin{gathered}
Q \sim L^{d} v^{(d+z) v /(z v+1)} g_{r}\left(v L^{1 / v+z}\right), \\
F \sim L^{d} v^{d v /(z v+1)} f_{r}\left(v L^{1 / v+z}\right),
\end{gathered}
$$

where $\quad g_{r}(x \ll 1) \sim x^{2-(d+z) v /(z v+1),} \quad f_{r}(x \ll 1) \sim$ $x^{2-d v /(z v+1)} \quad$ (very slow, i.e., LZ regime) and $g_{r}(x \gg 1) \sim$ const, $f_{r}(x \gg 1) \sim$ const $(\mathrm{KZ}$ regime $) .{ }^{19}$

The above-mentioned expectations are corroborated in Figs. 2(a) and 2(c), which show the behavior of $Q$ and $F$ as a function of the quench rate $v^{1}$ for several system sizes $10 \leqslant L \leqslant 96$ on a log-log scale. For both $Q$ and $F$ we find $v^{2}$ scaling for very slow ramps and KZ-like scaling $Q \sim v^{1}$ and $F \sim v^{1 / 2}$ for intermediate ramps. Finally, for fast ramps, we find non-power-law behavior corresponding to the breakdown of universality. ${ }^{22}$ The LZ-KZ scaling crossover [Eqs. (9) and (10)] is further corroborated in Figs. 2(b) and 2(d). These plots demonstrate both the scaling collapse of $Q$ and $F$ for slower ramps (points to the left) and their deviations from scaling for faster ramps (points to the right) in the nonuniversal regime. The dashed lines indicate the expected form of the scaling functions $g_{r}$ and $f_{r}$ in both the LZ and the KZ regimes. Note that for the LZ regime, we see collapse even for relatively small system sizes $(L \gtrsim 10)$. For the $\mathrm{KZ}$ regime, collapse only occurs for larger system sizes, extending roughly from $v \sim 70 / L^{2}$ up to $v \sim 0.5$.

Next, we study ramps that cross the QCP. As the system progresses toward the QCP, the gap decreases, and quasiparticle excitations are produced. After passing the QCP the gap increases, and the evolution eventually becomes adiabatic.
Assuming that the dynamics is dominated by the integrable behavior near the QCP, the number of quasiparticle excitations in the final adiabatic part of the ramp is conserved. In particular, for an ending point deep in the AFM phase, almost all excitations would be converted into domain walls with energy per excitation $\sim\left|U-\mathcal{E}_{f}\right|$. Therefore, one naively expects $Q$ to become proportional to the number of excitations, and hence scale the same way as $F$. These scaling expectations are corroborated for our nonintegrable model in Figs. 2(e) and 2(f), in which we plot $Q$ and $F$ for ramps from PM $\left(U-\mathcal{E}_{i}=\right.$ $100)$ to AFM phase $\left(U-\mathcal{E}_{f}=-100\right)$. Not only do we find KZ-like scaling in $F \sim v^{1 / 2}$ as expected, but $Q$ also scales as $v^{1 / 2}$. While our argument is hardly proof of the origin of "anomalous" exponents, we suggest that anomalous scaling of $Q$ in ramps across the QCP should be interpreted with caution.

Having obtained the scaling behavior, we concentrate on its manifestation for experimentally observable quantities. We note that the existing experimental setup ${ }^{7}$ focuses on imaging the parity of the number of bosons on each site, after projecting the wave function into a Fock state. . $^{3,4}$ Effectively, this imaging counts the number of empty and doubly occupied sites (i.e., "defects" on the PM side). In addition to measuring this dipole number $n_{d}$, the existing experiments can measure the dipole-dipole correlation function $g_{d}(\ell)=\left\langle n_{d}(i) n_{d}(i+\ell)\right\rangle \sim$ $\left\langle S_{i}^{z} S_{i+\ell}^{z}\right\rangle=C_{i, i+\ell}$ at the end of the ramp. In Fig. 3(a), we plot the dipole density $n_{d} / L$ as a function of the $U-\mathcal{E}_{F}$. We note that the final saturation value of $n_{d}$ in the AFM phase is a decreasing function of the ramp rate and lies between those for a nearly adiabatic ramp and a sudden quench. In Fig. 3(b), we show that the defect number $D$ demonstrates similar finite-sized KZ scaling as $F$. Since $n_{d}$ is experimentally measurable, our work demonstrates that finite-sized scaling 

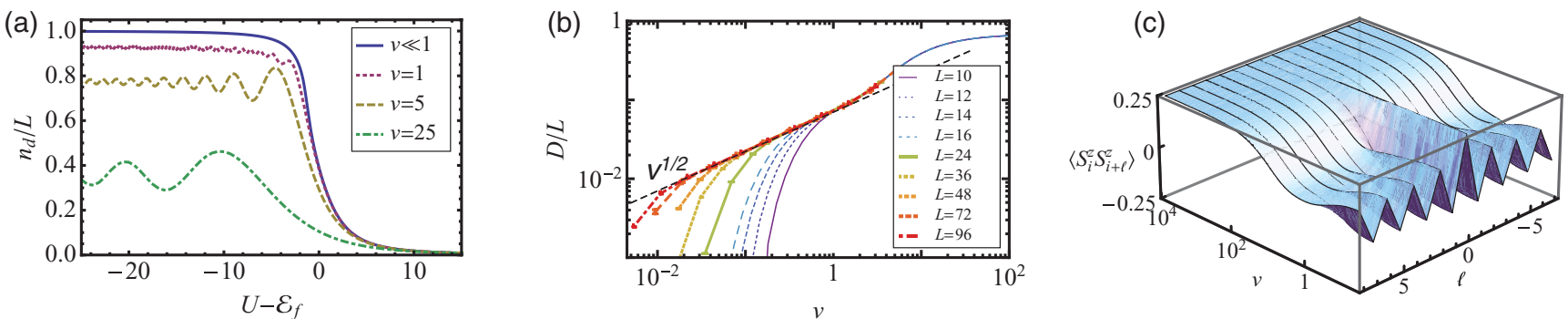

FIG. 3. (Color online) (a) $n_{d} / L$ as a function of the end point of the ramp $U-\mathcal{E}_{f}$ for several ramp rates (all ramps start in the PM phase $U-\mathcal{E}_{i}=50$ with $L=16$ ). (b) $D / L$ as a function of $v$ for several $L$ showing $\mathrm{KZ}$ scaling $\left(U-\mathcal{E}_{i}=100, U-\mathcal{E}_{f}=-1.31\right.$ ). (c) Spin-spin correlation function $C_{\ell}=\left\langle S_{i}^{z} S_{i+\ell}^{z}\right\rangle$ at the end of the ramp as a function of $\ell$ and the ramp rate $v$ for $L=16$.

can be observed within an existing experimental setup. Finally, in Fig. 3(c), we show the behavior of $C_{i, i+\ell}$ as a function of $v$ and $\ell$. We note that for slow ramps to the QCP one finds an oscillatory behavior of $C_{i, i+\ell}$, indicating the precursor of the AFM order present in the critical ground state. As $v$ is increased, the system ceases to reach the final ground state and these correlations decay. Finally, the state of the system at the end of fast ramps is essentially the PM ground state, which has no AFM correlations, leading to a flat $C_{i, i+\ell}$. These features can be directly picked up in experiments, which can serve as a test of our theory.

Beyond its intrinsic interest, we suggest two applications of our work. The first application is to provide an alternative view on how to probe universality. Traditionally, one would bring the system to equilibrium in the vicinity of a quantum critical point, and then probe it. Achieving equilibrium is particularly difficult in ultracold atom systems. Directly studying dynamical properties obviates the necessity of this equilibration. Since our results are at the horizon of what is experimentally achievable in cold atom experiments, they provide suggestions for experimentally probing dynamic critical phenomenon.

A second application is guidance for initializing quantum emulators to interesting quantum states; a quantum emulator will likely have to start with an well-known ground state and take the system through a QCP. Our method provides a direct estimate of the fidelity of this procedure for a nonintegrable system.
In conclusion, we have investigated the universal scaling dynamics of a finite-sized nonintegrable bosonic system following a finite rate ramp of the effective electric field. Our investigation demonstrates two scaling regimes (LZ- and KZ-like scaling) with conventional exponents and thus differs from prior studies of other nonintegrable systems ${ }^{1,14,17}$ which found various anomalous scaling exponents. Furthermore, comparing ramps that cross the QCP (which show anomalous exponents) to those that end at the QCP (which show expected exponents), we suggest a possible origin of these anomalous exponents: the adiabatic dynamics of the excitations following the passage through the quantum critical regime. Our work provides evidence of a real-time finite-sized scaling collapse of dynamics in nonintegrable systems, which we postulate should extend to other quantum phase transitions. Finally, we compute experimentally measurable quantities such as the dipole density $n_{d} / L$ and the dipole correlation function $C_{i j}$, and demonstrate that the scaling behavior studied in this work can be observed in realistic experiments.

It is our pleasure to thank A. Polkovnikov for invaluable discussions. We acknowledge support from the Lee A. DuBridge fellowship (D.P.), IIAS, PCTS. K.S. thanks DST, India for support through Grant No. SR/S2/CMP-001/2009. M.K. acknowledges support from ARO Award W911NF-071-0464 with funds from the DARPA OLE Program. D.P. and B.K.C. thank the Aspen Center for Physics for its hospitality.
${ }^{1}$ F. Pollmann, S. Mukerjee, A. G. Green, and J. E. Moore, Phys. Rev. E 81, 020101(R) (2010); J.-S. Bernier, G. Roux, and C. Kollath, Phys. Rev. Lett. 106, 200601 (2011).

${ }^{2}$ A. Polkovnikov, K. Sengupta, A. Silva, and M. Vengalattore, Rev. Mod. Phys. 83, 863 (2011); D. Ziarmaga, Adv. Phys. 59, 1063 (2010)

${ }^{3}$ M. Greiner, O. Mandel, T. Esslinger, T. W. Hansch, and I. Bloch, Nature (London) 415, 39 (2002).

${ }^{4}$ J. Simon, W. Bakr, R. Ma, M. E. Tai, P. Preiss, and M. Greiner, Nature (London) 472, 307 (2011).

${ }^{5}$ S. Sachdev, K. Sengupta, and S. M. Girvin, Phys. Rev. B 66, 075128 (2002).

${ }^{6}$ S. Pielawa, T. Kitagawa, E. Berg, and S. Sachdev, Phys. Rev. B 83, 205135 (2011).

${ }^{7}$ W. S. Bakr, A. Peng, M. E. Tai, R. Ma, J. I. Gillen, and S. Föllen, Science 329, 547 (2010).
${ }^{8}$ C. Trefzger and K. Sengupta, Phys. Rev. Lett. 106, 095702 (2011).

${ }^{9}$ K. Sengupta, S. Powell, and S. Sachdev, Phys. Rev. A 69, 053616 (2004).

${ }^{10}$ C. P. Rubbo, S. R. Manmana, B. M. Peden, M. J. Holland, and A. M. Rey, Phys. Rev. A 84, 033638 (2011).

${ }^{11}$ A. Tomadin, R. Mannella, and S. Wimberger, Phys. Rev. A 77, 013606 (2008)

${ }^{12}$ A. R. Kolovsky, Phys. Rev. A 70, 015604 (2004).

${ }^{13}$ There are two minor differences between our setup and current experimental setups: (1) We use periodic boundary conditions while experiments have thus far used open boundary conditions; (2) experiments have a small amount of disorder while we study clean systems.

${ }^{14}$ T. W. B. Kibble, J. Phys. A 9, 1387 (1976); W. H. Zurek, Nature (London) 317, 505 (1985). 
${ }^{15}$ A. Polkovnikov and V. Gritsev, Nat. Phys. 4, 477 (2008); C. De Grandi and A. Polkovnikov, in Quantum Quenching, Annealing and Computation, edited by A. Das, A. Chandra, and B. K. Chakrabati, Lecture Notes in Physics, Vol. 802 (Springer, Heidelberg, 2010).

${ }^{16}$ M. M. Rams and B. Damski, Phys. Rev. Lett. 106, 055701 (2011).

${ }^{17}$ A. Polkovnikov, Phys. Rev. B 72, 161201(R) (2005); R. W. Cherng and L. S. Levitov, Phys. Rev. A 73, 043614 (2006); C. De Grandi, V. Gritsev, and A. Polkovnikov, Phys. Rev. B 81, 224301 (2010).

${ }^{18}$ N. V. Vitanov and B. M. Garraway, Phys. Rev. A 53, 4288 (1996).
${ }^{19}$ C. De Grandi, A. Polkovnikov, and A. W. Sandvik, Phys. Rev. B 84, 224303 (2011)

${ }^{20}$ U. Schollwock, Ann. Phys. 326, 96 (2011); G. Vidal, Phys. Rev. Lett. 91, 147902 (2003).

${ }^{21}$ To speed up tMPS, for slower ramp rates we move the starting point $U-\mathcal{E}_{i}$ closer to the QCP. We have verified that this does not affect the observables.

${ }^{22}$ Care must be taken in interpreting data on smaller systems, as these appear to show a small segment of anomalous power-law-like scaling in the $\mathrm{KZ}$ regime. However, this behavior is a finite-sized effect and the universal $\mathrm{KZ}$ exponents are restored for larger system sizes. 